\title{
UV Laser Photo-Polymerization of Elastic 2D/3D Structures Using Photo-Curable PDMS (Polydimethylsiloxane)
}

\author{
Oliver Suttmann*, Kotaro Obata*, Yasutaka Nakajima**, Arndt Hohnholz*, Jürgen Koch*, \\ Mitsuhiro Terakawa**,***, and Ludger Overmeyer* \\ ${ }^{*}$ Laser Zentrum Hannover e.V., Hollerithallee 8, 30419 Hanover, Germany \\ Email: o.suttmann@lzh.de \\ ** School of Integrated Design Engineering, Keio University, 3-14-1 Hiyoshi, Kohoku-ku, Yoko- \\ hama, 223-8522, Japan \\ *** Department of Electronics and Electrical Engineering, Keio University, 3-14-1, Hiyoshi, Ko- \\ hoku-ku, Yokohama, 223-8522, Japan
}

\begin{abstract}
2D/3D structuring of UV curable polydimethylsiloxane (PDMS) was developed using UV laser direct writing. In this technique, commercially available UV curable PDMS was locally polymerized to fabricate 1D and 2D single layer structures, as well as 3D multi-layer structures with sufficient stability in process resolutions. The tightly focused UV laser beam was scanned by an $\mathrm{x}-\mathrm{y}$ galvanometric mirror scanner on the UV curable PDMS layer coated on the glass substrate, so that 18$\mu \mathrm{m}$ of process resolution in X-Y plane was obtained. At the relevant wavelength of $355 \mathrm{~nm}$, the absorbance of UV curable PDMS was 0.131, which is sufficient absorbance for a photo-chemical reaction induced by the UV laser used in this study. In addition, UV curable PDMS has a deep penetration depth, longer than $3 \mathrm{~mm}$, which enabled the fabrication of a structure with an aspect ratio of 3 in a single layer. Furthermore, the fabricated structure demonstrated mechanical strength to emphasize its elasticity.
\end{abstract}

DOI: $10.2961 /$ jlmn.2017.02.0018

Keywords: UV curable PDMS, UV laser, laser direct writing, photo-polymerization, additive manufacturing

\section{Introduction}

The micro/nano structuring of polydimethylsiloxane (PDMS) is a useful technique not only for research, but also for industrial applications. For structuring using thermal curing type PDMS, the mold technique is established as the major process [1,2]. On the other hand, a combination of photo-curable type PDMS and ultra-short pulse laser has been demonstrated as another approach to micro/nano structuring of PDMS [3-7].

Recently, the cost performance of high power UV light sources has improved with advancements in technology. As a result of this improvement, commercially available UV curable polydimethylsiloxane (PDMS) has been newly released and has received a surge of attention as an alternative for conventional thermal curing types of PDMS [8]. The commercially available UV curable PDMS is designed for photo-polymerization at UV wavelengths, which makes it a suitable material for additive manufacturing by UV laser direct writing (UV-LDW). In UV-LDW of UV curable PDMS, a tightly focused UV laser beam scans the UV curable PDMS, resulting in localized photo-polymerization with free-form shapes. In this paper, we demonstrate the fabrication of single layered 1D/2D and multi-layered 3D structures of UV curable PDMS by UV-LDW.

\section{Experimental procedure}

Figure 1 shows a schematic illustration of the experimental setup for laser direct writing of UV curable PDMS.
In this experiment, a UV curable PDMS (KER-4690 A/B, Shin-Etsu Silicones Europe B.V.) was prepared with a premixed condition of the two components (50/50 wt.\% mixture of A and B) before laser irradiation. The UV curable PDMS layers with various thickness were created by spin coating for layers thinner than $100 \mu \mathrm{m}$ or by drop casting for layers in millimeters scale. For the photo-

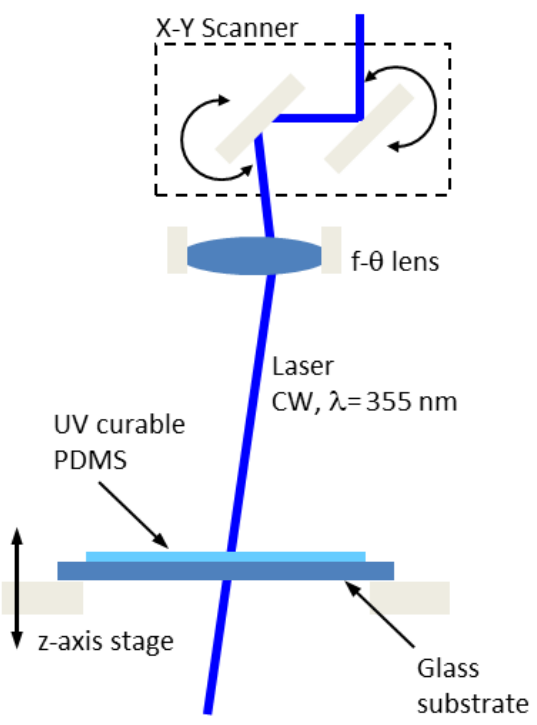

Fig. 1 Schematic illustration of the experimental setup for 2D/3D structuring using UV curable PDMS 
polymerization process, a continuous wave UV laser system (Cobolt AB, Model Zouk ${ }^{\mathrm{TM}}$, CW 20 mW, $355 \mathrm{~nm}$ ) was used as a light source. Timing of the laser irradiation and laser power were adjusted by an acousto-optic modulator (AOM) and neutral density (ND) filters. The laser beam was scanned by an $\mathrm{x}-\mathrm{y}$ galvanometric mirror scanner (Hurry SCAN 14, SCANLAB AG) and positioned in the z-axis by a high-resolution linear stage (MT105-50 LM, Feinmess Dresden $\mathrm{GmbH}$ ). The motion of the scanner for 2D patterning with laser irradiation was controlled by SAMLight (SCAPS) software and CAD data. The laser beam was focused on the sample surface with a $103 \mathrm{~mm}$ focal-length FTheta lens. After laser irradiation, the structures are not sufficientlly polymerized for the development process. Thus, the fabricated structures were treated by post-thermal treatment for $10 \mathrm{~min}$ at $50{ }^{\circ} \mathrm{C}$ to accelerate the polymeriation only at the laser exposured areas. The samples with enhanced polymerization were then developed with tetrahydrofuran (THF).

\section{Results and discussion}

For 2D patterning of a single layer of the UV curable PDMS, controllable layer thickness is an important parameter in achieving structuring using UV-LDW. The thickness of the UV curable PDMS layer was controlled by parameters in the spin-coating process. Figure 2 (a) shows the dependence of the layer thickness on the spin-coating rotation speed. In the spin-coating process, droplets of UV

(a)

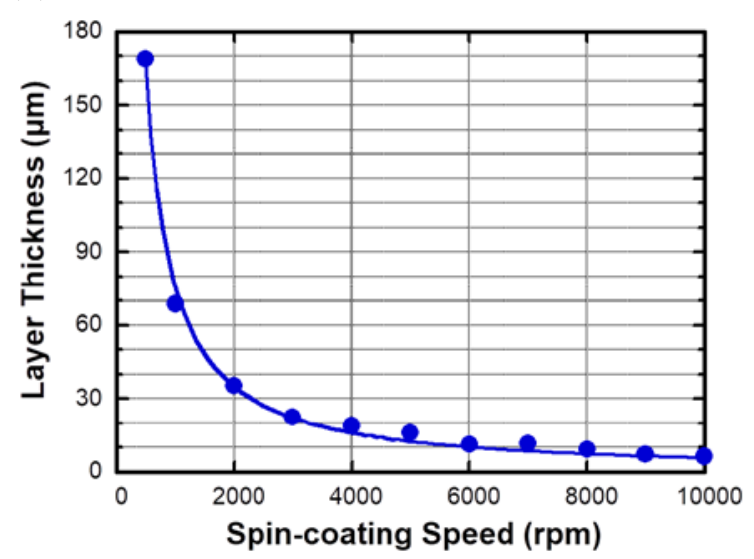

(b)

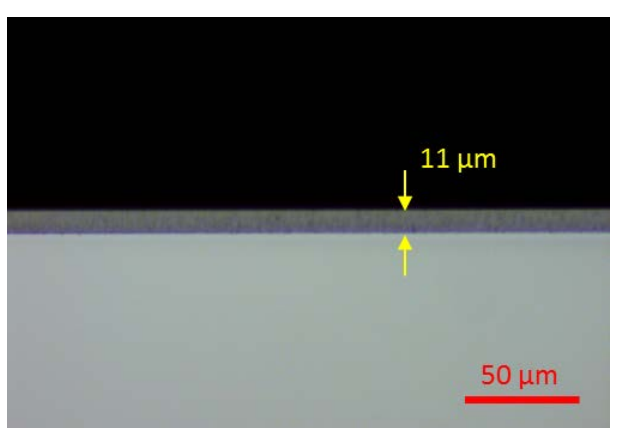

Fig. 2 (a) dependence of the layer thickness on the spin-coating rotation speed, (b) cross sectional image of coated UV curable PDMS layer coated at spin-coating speed of $6000 \mathrm{rpm}$ for $30 \mathrm{~s}$. curable PDMS were spun on glass substrates at different rotation speeds for $30 \mathrm{~s}$. The coated layers of the samples were photo-polymerized under a UV LED lamp (Dymax BlueWave ${ }^{\circledR}$, Model QX44 ${ }^{\mathrm{TM}}$, RediCure ${ }^{\mathrm{TM}} 365 \mathrm{~nm}$ ) after coating and then put into an oven at $155^{\circ} \mathrm{C}$ for 15 min to accelerate polymerization. After the polymerization, the cross-sectional images of the samples were viewed under the optical microscope (Leica Microsystems Switzerland) and the layer thickness was measured. The thickness of the coated layer decreased with increasing rotation speeds. Figure 2 (b) shows a cross sectional image of a UV curable PDMS layer coated at spin-coating speed of $6000 \mathrm{rpm}$ for $30 \mathrm{~s}$. The morphology of the coated layer indicated uniform thickness of $11 \mu \mathrm{m}$ and adhesion to the glass substrate.

Figure 3 shows the absorbance spectrum of the UV curable PDMS for wavelengths of $320 \mathrm{~nm}$ to $610 \mathrm{~nm}$. The absorbance spectrum was obtained from transmittance spectrum on a $5 \mathrm{~mm}$ thick sample measured with a spectrometer (UV-1650 PC, Shimadzu Europe). The UV curable PDMS has little absorbance in the visible range, and exhibits an absorption range of wavelengths shorter than $455 \mathrm{~nm}$. At the relevant wavelength of $355 \mathrm{~nm}$, the absorbance was 0.131 , which is sufficient absorbance for a photochemical reaction in volume.

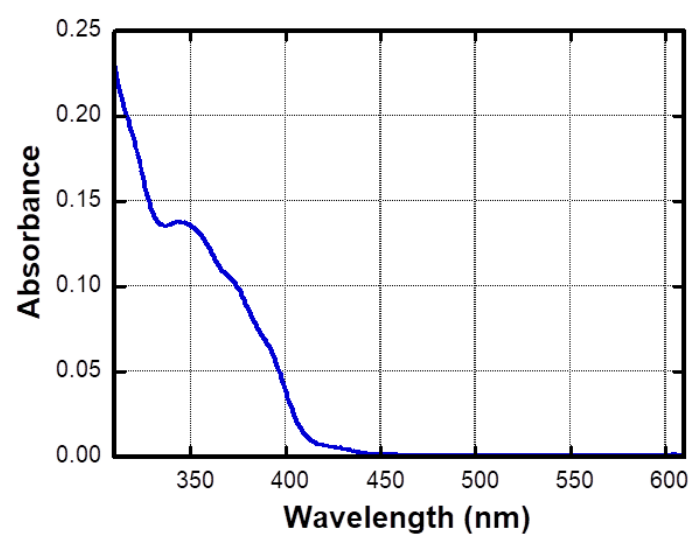

Fig. 3 Absorbance spectrum of UV curable PDMS

As an example of the UV curable PDMS fabricated by UV-LDW. Figure 4 shows the polymerization of line arrays for line widths of $29 \mu \mathrm{m}$ with a pitch of $230 \mu \mathrm{m}$. Before laser irradiation, UV curable PDMS was spin coated onto a glass substrate at $4000 \mathrm{rpm}$ for $60 \mathrm{~s}$, creating a layer thickness of $14 \mu \mathrm{m}$. Each line was produced at a laser power of $4.5 \mathrm{~mW}$ and laser scanning speed of $500 \mathrm{~mm} / \mathrm{s}$, with 750 laser irradiation cycles. The lines are sharp and uniform in their widths, height, and smoothness, indicating repeatable and stable polymerization of the UV curable PDMS. There is no distortion in the lines, showing physical stability to the development process. 


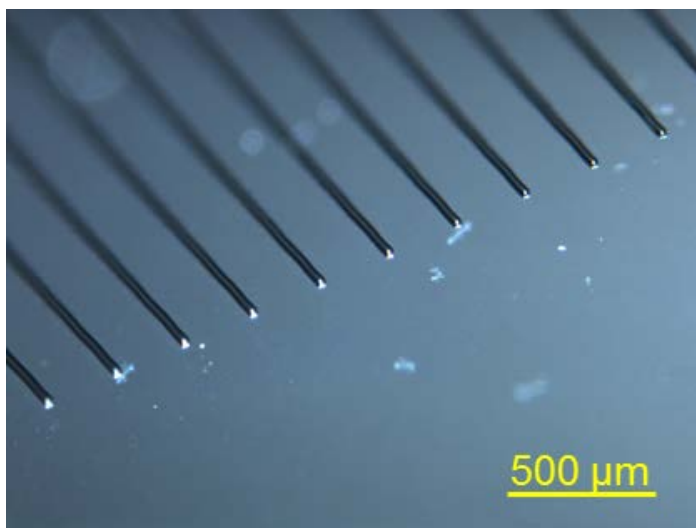

Fig. 4 Line array with line widths of $29 \mu \mathrm{m}$ and a pitch of $230 \mu \mathrm{m}$

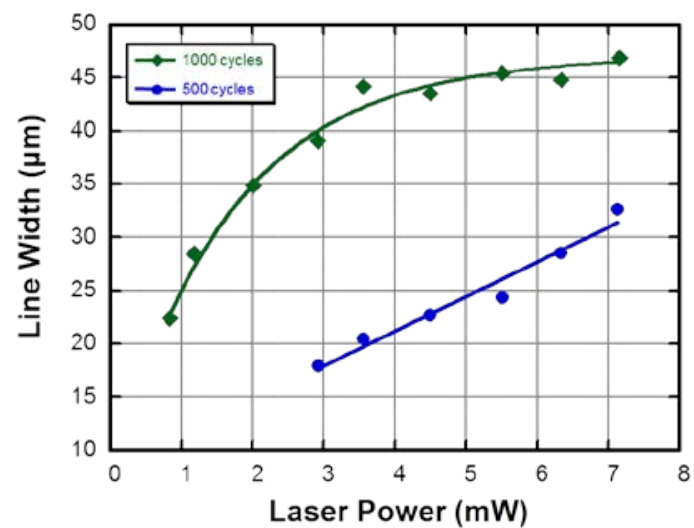

Fig. 5 Dependence of line width on input laser power

The height of fabricated line array structure shown in Fig. 4 is consistent with the thickness of the spin coated UV curable PDMS. The dependence of line width on input laser power is shown in Fig. 5. The line arrays were fabricated at a laser scanning speed of $500 \mathrm{~mm} / \mathrm{s}$ with 500 or 1000 laser irradiation cycles. Line widths between $18 \mu \mathrm{m}$ and $47 \mu \mathrm{m}$ were produced using different laser powers and laser scanning speeds. The line width measurement data from all line array patterns, shown in Fig. 5, demonstrates the correlation between laser power and X-Y resolution of polymerized structures. The findings consistently show that an increase in laser power leads to an increase in line widths.

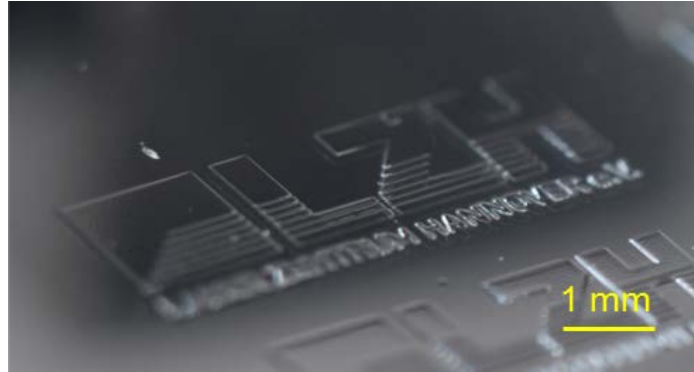

Fig. 6 LZH logo produced with 2D patterning in UV curable PDMS by UV-LDW
Figure 6 shows the LZH logo produced with 2D patterning of UV curable PDMS by UV-LDW. The structures were $6.00 \mathrm{~mm}$ by $2.23 \mathrm{~mm}$ with a thickness of $14 \mu \mathrm{m}$. UV curable PDMS was coated on a glass substrate by spincoating. The laser then irradiated the material with a scanning speed of $500 \mathrm{~mm} / \mathrm{s}$ and a laser power of $843 \mu \mathrm{W}$ with 20 laser irradiation cycles. Finally, THF was used to develop the sample. The fabricated structures had smooth surface which maintained transparency due to low surface roughness in the visible range, and sharp corners and edges. The scanned surface displayed good adhesion between hatch lines in both planer directions and no seam lines were
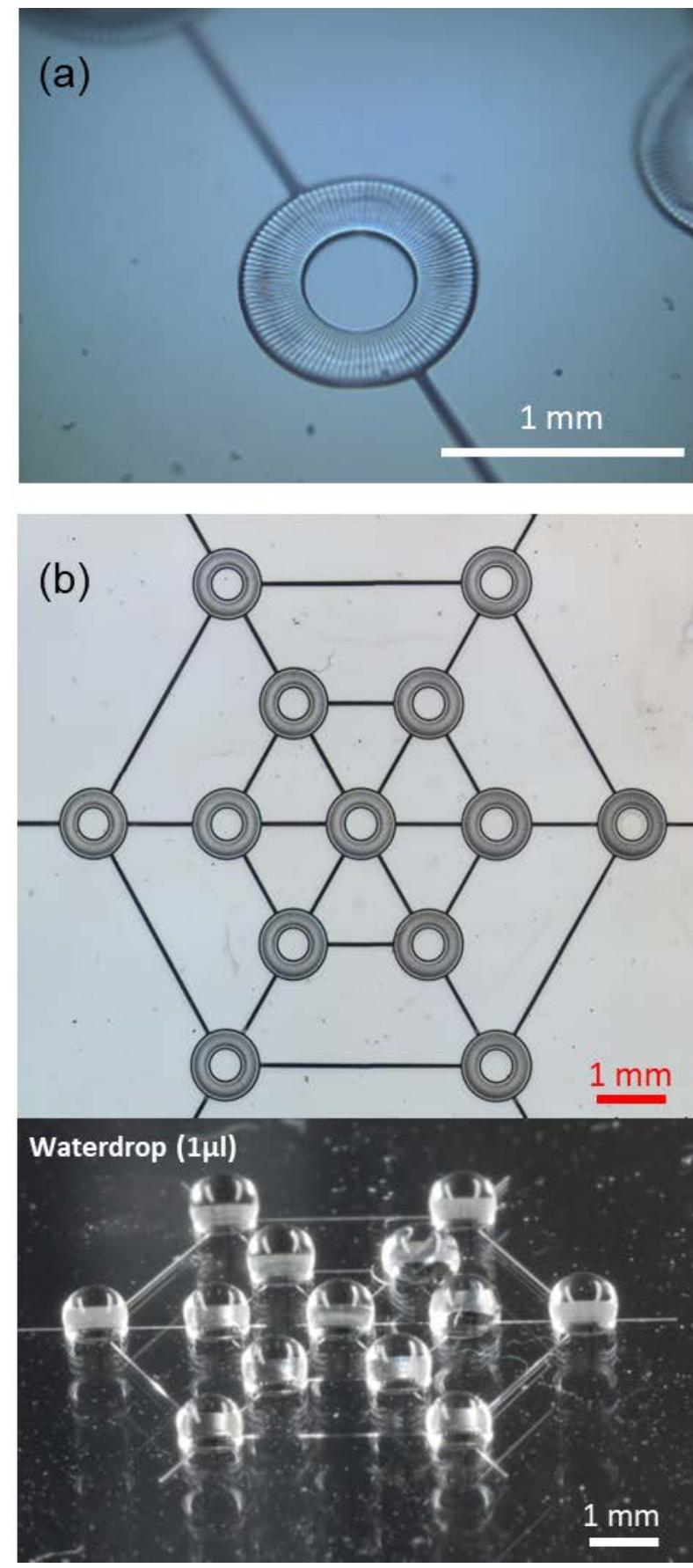

Fig.7 (a) ringstructure of UV curable PDMS, (b) a microarray of ring structures (Top), and $1 \mathrm{ul}$ water droplets deposited by pipette control (Bottom) 
visible in the direction of scanning. Localized curing was demonstrated by the ability to maintain thin gaps between in the bottom portion of the logo with sufficient contrast between polymerized and un-polymerized areas. Moreover, none of the samples showed deformations due to shrinkage effects. While this sample demonstrated 2D patterning with a material thickness of $14 \mu \mathrm{m}$. There is, in the future, the potential to control the film thickness. Films as thin as 1 um in thickness can be achieved in a combination with aerosol jet printing technology [9], while films thicker than $100 \mu \mathrm{m}$ can be made by drop casting. Use of the drop casting technique has been demonstrated in Fig. 8 and Fig. 9, later. Furthermore, there is the possibility of increasing throughput by the use of digital light projectors (DLP) for industrial applications.

As one potential application of $2 \mathrm{D}$ patterning by the localized polymerization of UV curable PDMS, Fig. 7 (a) shows ring shape structure of UV curable PDMS. The ring structures shown have an outer diameters of $1 \mathrm{~mm}$, inner diameters of $0.5 \mathrm{~mm}$, and thicknesses of $14 \mu \mathrm{m}$. Scanning of the ring structures was done in a circular hatching pattern. Periodic ripples are visible on the surface of the ring structure. This is attributed to the motion of the laser scanning. For the fabrication of ring structure, each circle is constructed from 100 straight line segments in the program of the laser scanning. The acceleration and deceleration of the laser during direction changes causes some areas to have longer exposure times than others. Due to the uneven distribution of laser energy, some areas suffer from overpolymerization. As a result, the overpolymerized areas obtained larger polymerization volumes than the other areas in the ring structure, resulting in the formation of the ripple structures. Figure 7 (b) shows a micro-array of ring structures which can be used for position control of liquid droplets. The image on the bottom shows $1 \mu$ l water droplets, deposited by pipette control, resting on the rings. Selfalignment of the water droplets to the ring structure is achieved due to the hydrophobic properties of the PDMS surface.

The polymerization of thick layers prepared by drop casting technique was demonstrated. Figure 8 (a) shows rectangular pillar array structure fabricated by $2 \mathrm{D}$ patterning of UV-LDW. Using the drop casting technique, $1 \mathrm{~mm}$ of PDMS material was dropped into a $15 \mathrm{~mm}$ diameter circular frame. The frame itself had a height of $3 \mathrm{~mm}$. the UV laser scanned an array of $300 \mu \mathrm{m}$ by $100 \mu \mathrm{m}$ rectangles onto the dropped material and then developed in THF. This was done with laser scanning of $1000 \mathrm{~mm} / \mathrm{s}$ at a power of $1.75 \mathrm{~mW}$ with 20 laser irradiation cycles. This sample demonstrates the successful fabrication of self-standing, higher than 3 in aspect ratio structures; surfaces are smooth, and edges and corners are sharp. The material also shows good elasticity.

Figure 8 (b) shows the letters LZH made using the same method as the pillars in Fig. 8 (a). In this case, the structures were removed from the glass substrate after development and placed upright. In order to make removal of the structure from the glass substrate possible, the laser power, scanning speed, and irradiation cycles were adjusted to $843 \mu \mathrm{W}, 1000 \mathrm{~mm} / \mathrm{s}$ and 20 cycles which are nearly equal to polymerization threshold. When a part of the structure was bent, the structure did not break from defects. This (a)
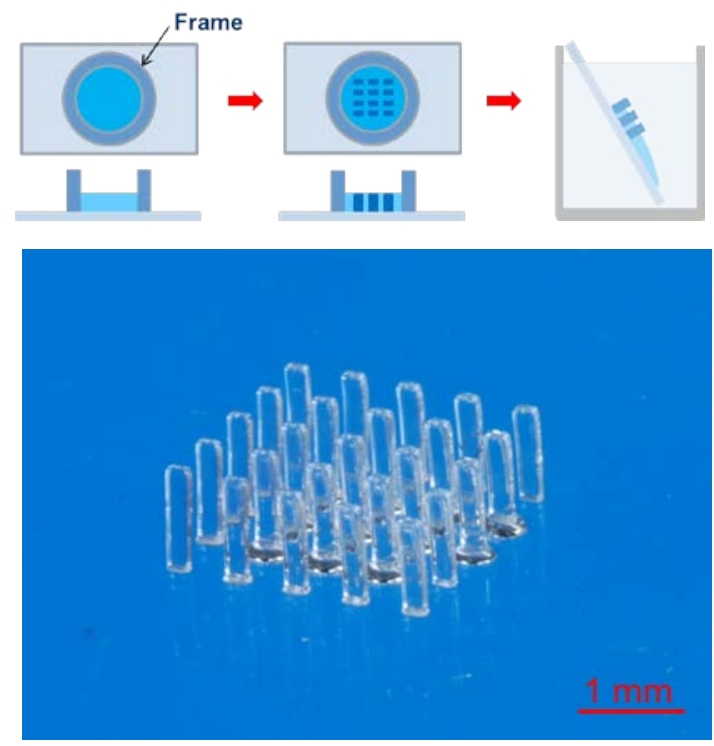

(b)

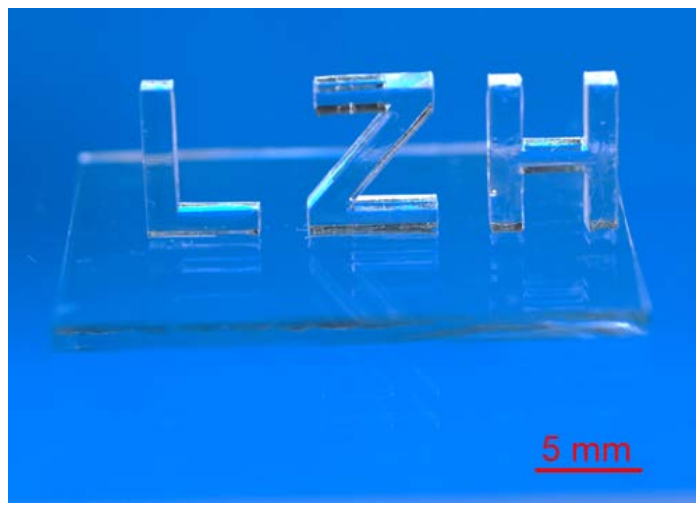

Fig. 8 (a) rectangular pillar array structure fabricated by 2D patterning of UV-LDW, (b) the letters LZH

reveals that the structures exhibited sufficient mechanical strength and elasticity. In addition, the surface of the structure had good transparency and smoothness, as the edge of the glass substrate behind the structure can be seen without distortion. The structures also had smooth, flat surfaces for both the laser exposed area and the boundaries between polymerized and unpolymerized material. The sharp edges and corners demonstrate good contrast in photopolymerization. Furthermore, using the method of 2D scanning in drop cast UV curable PDMS, a penetration depth of the laser beam deeper than $5 \mathrm{~mm}$ has been achieved.

Demonstration of 3D structuring was done with the creation of a spider web style net, illustrated in Fig. 9 (a). Each layer of liquid UV curable PDMS was dropped into a circular frame used in Fig. 8 on a glass substrate. The circular frame keeps the required layer thickness of UV curable PDMS. The dropped UV curable PDMS layer was irradiated at a laser power of $843 \mu \mathrm{W}$ and a laser scanning speed of $1000 \mathrm{~mm} / \mathrm{s}$ in a spider web pattern. A second layer was then dropped in the frame and irradiated again at a laser power of $843 \mu \mathrm{W}$ and a laser scanning speed of 1000 $\mathrm{mm} / \mathrm{s}$ to create the part of posts. Thus, the entirety of the $3.6 \mathrm{~mm}$ structure could be built in a two layer process, 
(a)
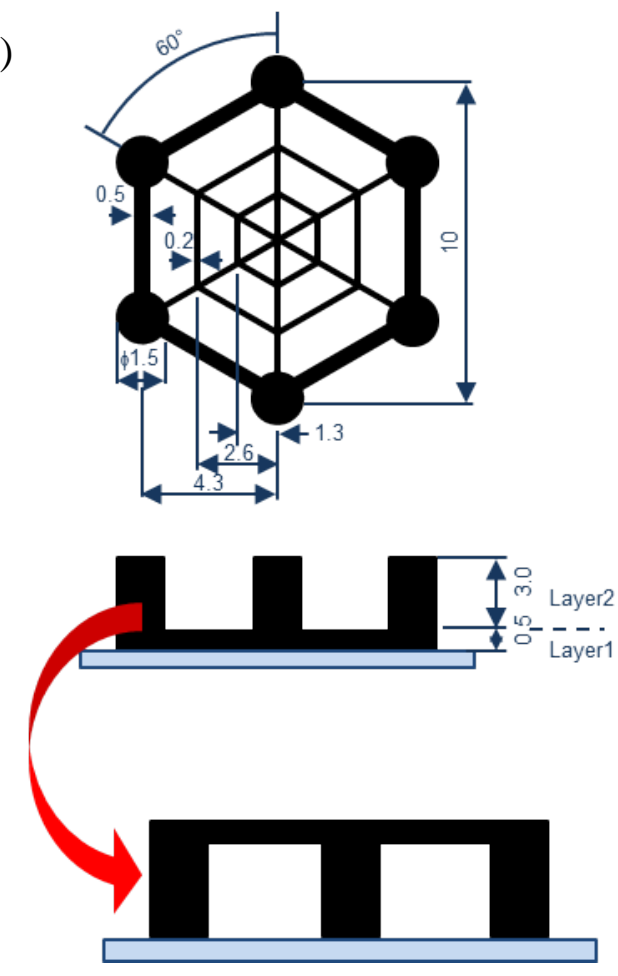

(b)

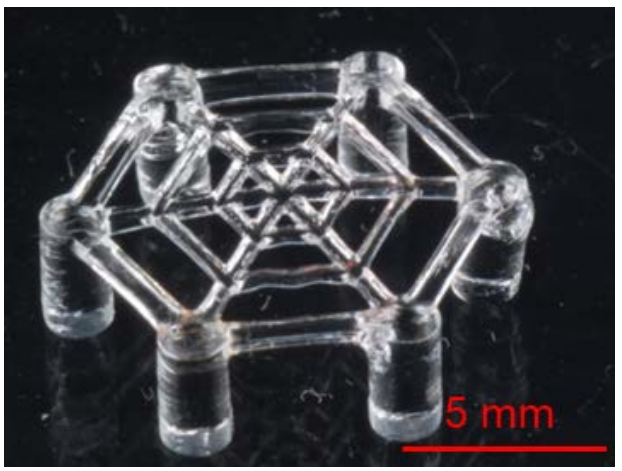

(c)

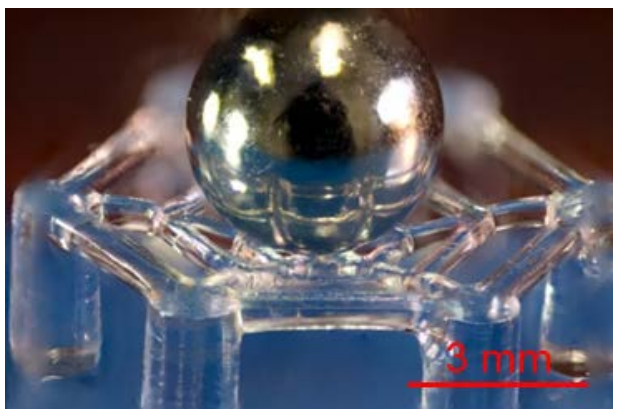

Fig. 9 (a) a design of spider web style net, (b) overview of the multi-layer spider web style net structure, (c) multi-layer spider web style net structure holding steel ball

demonstrating the high aspect ratio of the UV curable PDMS. The discrepancy between the technical drawings measurements of the net and the actual measurements, about $200 \mu \mathrm{m}$, was due to the width of the laser beam, and can be adjusted by the laser exposure conditions. The difference in height, $100 \mu \mathrm{m}$ was due to the amount of material deposited in the frame. After post treatment, the structure was turned over so the posts supported the full weight of the web. The overview of the fabricated structure is shown in Fig. 9 (b). The structure was again able to maintain its transparency in the visible range and had smooth outer surfaces. Due to the absorption depth of the UV curable PDMS shown in Fig. 8 as well, a $3 \mathrm{~mm}$ thick layer can be polymerized without a layer by layer process. Thus, pillars on the web structure with an aspect ratio of 2 could be obtained. The polymerized PDMS was mechanically strong and flexible, even in the thinnest $0.39 \mathrm{~mm}$ section. Figure 9 (c) shows a $7 \mathrm{~mm}$ diameter, $1.49 \mathrm{~g}$ steel ball that was held in the netting of the structure shown in Fig. 9 (b). The surface of the web structure sunk $0.6 \mathrm{~mm}$ when the steel ball was loaded on the web. The pillar structures were not bent due to tensile stress from the web part. This reveals that the strength of the pillar structures and elasticity of the web could suspend the steel ball above the glass substrate. The netting was able to return to its original shape after the steel ball was removed.

\section{Conclusion}

In conclusion, the fabrication of 2D/3D structuring using an UV curable PDMS has been demonstrated by UV laser direct writing. Structures of UV curable PDMS were obtained with a lateral resolution of $18 \mu \mathrm{m}$. The fabricated structures had sufficient physical strength to show elasticity of UV curable PDMS. In addition, the structure maintained a transparent appearance and smoothness. The development process caused little shrinkage and did not induce obvious deformations in surface morphology. The UV curable PDMS used in this experiment had a sufficient absorbance of 0.131 and an absorption depth longer than $3 \mathrm{~mm}$ to the UV laser beam. This long absorption depth enabled the achievement of a high-aspect ratio of 3 , by curing in a single step of laser irradiation. The laser direct writing process can achieve maskless patterning of UV curable PDMS. Thus, these new approaches using UV curable PDMS are promising for wide and more flexible applications in additive manufacturing.

\section{Acknowledgments}

Authors would like to thank Shin-Etsu Silicones Europe B.V. for providing the PDMS sample and Katie Xu for supporting the technical discussion. This research was supported in a part of joint research projects by "Projektbezogener Personenaustausch mit Japan” (DAAD-JSPS) Joint Research Program (Project 57245147). The authors acknowledge financial support in the frame of the 3DPolySPRINT Project (BMBF FKZ 13N13567).

\section{References}

[1] Y. Lin and J. Chou, J. Adhes: Sci. Technol., 30, (2016) 1310.

[2] T. Nargang, L. Brockmann, P. Nikolov, D. Schild, D. Helmer, and N. Keller: Lab Chip, 14, (2014) 2698.

[3] C. Coenjarts and C. Ober: Chem. Mater. 16, (2004) 5556.

[4] T. Hasegawa, K. Oishi, and S. Maruo: Proc. of IEEE Conference on Micro-NanoMechatronics and Human Science (MHS), Nagoya, Japan, (2006) p.1.

[5] H. Selvaraj, B. Tan, and K. Venkatakrishnan: J. Micromech. Microeng., 21, (2011) 075018. 
[6] S. Rekštytè, M. Malinauskas, and S. Juodkazis: Opt. Express, 21, (2013) 17028.

[7] C. Sones, I. Katis, B. Mills, M. Feinaeugle, A. Mosayyebi, J. Butement, and R. Eason: Appl. Surf. Sci., 298, (2014) 125.

[8] N. Chidambaram, R Kirchner, M Altana, and $\mathrm{H}$. Schift: J. Vac. Sci. Technol. B, 34, (2016) 06K401.

[9] K. Obata, U. Klug, J. Koch, O. Suttmann and L. Overmeyer: JLMN, 9, (2014) 242.

(Received: June 30, 2017, Accepted: September 10, 2017) 